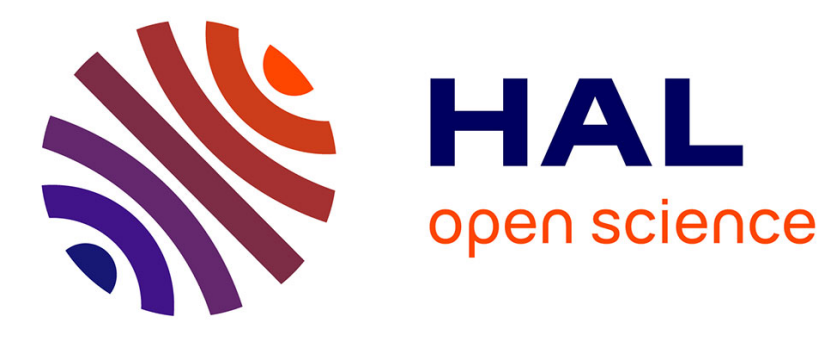

\title{
A generic and modular architecture for maritime autonomous vehicles
}

Magali Barbier, Eric Bensana, Xavier Pucel

\section{To cite this version:}

Magali Barbier, Eric Bensana, Xavier Pucel. A generic and modular architecture for maritime autonomous vehicles. 2018 IEEE OES Autonomous Underwater Vehicle Symposium AUV2018, Nov 2018, PORTO, Portugal. hal-01983412

\section{HAL Id: hal-01983412 \\ https://hal.science/hal-01983412}

Submitted on 16 Jan 2019

HAL is a multi-disciplinary open access archive for the deposit and dissemination of scientific research documents, whether they are published or not. The documents may come from teaching and research institutions in France or abroad, or from public or private research centers.
L'archive ouverte pluridisciplinaire HAL, est destinée au dépôt et à la diffusion de documents scientifiques de niveau recherche, publiés ou non, émanant des établissements d'enseignement et de recherche français ou étrangers, des laboratoires publics ou privés. 


\title{
A generic and modular architecture for maritime autonomous vehicles
}

\author{
Magali BARBIER, Eric BENSANA, Xavier PUCEL \\ ONERA The French Aerospace Lab, FRANCE
}

\{Firstname.Lastname\}@onera.fr

\begin{abstract}
Autonomous systems face the challenge of managing disruptive events such as internal alterations, mission updates and environmental perturbations that always occur in an operational context. Autonomous vehicles must embed the capabilities to supervise their behaviour and to detect and react to such events. The complexity increases with the heterogeneity of vehicles in the team and the communication constraints. This paper describes the Robot System Onboard Architecture (RSOA) software developed in the SWARMs project for the achievement of offshore maritime operations. This generic and modular architecture implements high level capabilities in a semiautonomous or autonomous manner, and is deployed at a lower level on a heterogeneous swarm of vehicles. Target vehicles include autonomous and teleoperated underwater vehicles, and surface vehicles. Simulation and experimentations achieved on the Black Sea in Romania (July 2017) then in the Trondheim fjord in Norway (June 2018) highlighted the good performance of the RSOA.
\end{abstract}

Keywords - Autonomous robots, Maritime scenarios, Software architecture, Mission online adaptation.

\section{I.INTRODUCTION}

The extended use of uninhabited underwater and surface vehicles in offshore maritime operations is a solution to increase the safety of tasks previously assigned to divers and reduce operational costs.

The objective of the SWARMs H2020 project (2015-2018) is to allow the cooperation in these offshore missions of autonomous uninhabited vehicles having complementary capabilities: measurements by submarine vehicles (AUV Autonomous Underwater Vehicle), surveillance, relay and measurements by surface vehicles (USV Uninhabited Surface Vehicle) and intervention by underwater teleoperated vehicles (ROV Remotely Operated Vehicle).

The project approach is to design and develop an integrated platform that allows these heterogeneous vehicles to achieve the different phases of a mission. The SWARMs system is then composed of three components:

- A centralized Mission Management Tool (MMT) located inside the Command and Control Station for the generation of missions, the assignment of tasks to robot systems and the supervision of missions;

- A distributed MiddleWare system (MW) to ensure the information exchange between the MMT and vehicles through the Wi-Fi and acoustic communication channels, as well as information processing such as recording data in the data base, managing the embedded ontology etc.

- Several robot systems with their sensors for the achievement of the tasks.

This paper deals with the third item and more precisely with the development of a generic and modular software architecture decentralized in the vehicles that allows the team of robots to react autonomously and in real time to disruptive events such as partial or total sensor failure, loss of propulsion, or high currents.

This architecture named RSOA (Robot System Onboard Architecture) is based on the ROS middleware (Robot Operating System) and is connected on the one hand to the MMT by RF/Wi-Fi (vehicles on the surface) or acoustic (vehicles under the water) communications and on the other hand to the robot platforms of each vehicle (AUV, USV, ROV). The communications between vehicles and the mission control use the DDS protocol (Data Distribution Service), OpenSplice DDS Community being used in vehicles and TwinOaks CoreDX DDS solutions being used in the MW.

This architecture has been implemented on several robots of the project, validated by simulation, then simulation with hardware in the loop, and finally by experimentations at sea. Because of the complexity and the relevant risks of creating real events during a mission, some functionalities were only evaluated by simulation.

Section II presents the RSOA architecture. Sections III and IV respectively describe experimentations and simulations tests performed during the first demonstration event in Mangalia (Romania) in July 2017 then during the second and final demonstration planned in Trondheim (Norway) in June 2018. Section V concludes this work.

\section{THE ROBOT SySTEM ONBOARD ARCHITECTURE}

\section{A. Integration in the SWARMs System}

The SWARMs offshore scenarios discussed with end users allowed the definition of generic high level tasks: transit to a given point or area, survey of a given area, inspection of an object or a structure and pick up of an object. These tasks are then decomposed in a set of motion, perception, manipulation and communication generic actions: go to a waypoint, follow a row, follow a structure, inspect an area, acquire sensor data...

In the MMT, the mission defined by the human operator is planned and the tasks are assigned to available vehicles so as to minimize the time or cost for executing the mission. MMT planners are also capable of mapping high level tasks into 
detailed actions for vehicles that do not have onboard planners. For the other vehicles, a mission made of a sequence of high level tasks is directly sent by acoustic communication to each vehicle, and the onboard planner produces a detailed plan of actions from these tasks. In all cases, the architecture must allow the supervision of the achievement of the actions and their monitoring. When a disruptive event occurs, either the problem is treated at the vehicle level (computation of a new local plan) or the information is sent to the MMT for global treatment at the team level.

In general, AUVs need to achieve a good level of autonomy because of the communication constraints under water: this is the primary purpose of embedding the RSOA in them. USVs can also embed the RSOA even if communications with the MMT are less constrained. ROVs are not here only as a mechanical extension of the human operator: modern ROVs can be thought of as semi-autonomous devices receiving high level tasks from the MMT. In the SWARMs project, a specific version of the RSOA is embedded in the local control station of the ROV; either a human operator or a filtering process is in the loop.

The robot system sends to the MMT real time information on the achievement of the tasks/actions (completed, aborted, or failed), on data relevant to the mission (i.e. sensor data such as salinity, depth)... This allows the MMT to react in a centralized way and at team level if required. When the RSOA can manage the adaptation of the robot in its mission, the reaction will be local if possible.

The bidirectional connection described in the two previous paragraphs is achieved through the MW thanks to a specific RSOA module named ROS-DDS Proxy.

\section{$B$. Connection with the existing robots}

Robot capabilities (limits of the operational domain, tasks and actions that the robot can perform, reactions to specific events...) are described in a Robot Configuration file. This file is read when the RSOA starts and configuration file is stored in the ROS parameters in a structured way, making it available for each module of the RSOA.

To keep the genericity of tasks and actions and to take into account the various SWARMs vehicles, the connection with existing software of one robotic platform has been implemented through two modules:

- The Robot Generic Interface module (RGI) translates the generic actions into generic commands and stores situation, status and environmental data received from the Robot Specific Interface;

- The Robot Specific Interface module (RSI) is connected in a bidirectional way with the Robot Generic Interface. It translates generic commands into robot specific commands and collects data, events and faults of the robot; this module was developed by vehicle providers with the help of RSOA developers.

\section{Supervisor function}

The Robot Supervisor module is central in the RSOA and based on a model of the expected behaviour of vehicles in nominal situation and when disruptive events occur. It receives a set of tasks or actions from the MMT; when tasks are received, the supervisor interacts with the Robot Planner function to obtain the set of actions to be executed.

In nominal situation, the supervisor executes the actions and monitor their execution; it then interacts with the Robot Generic Interface for actions dispatching and status reporting.

When an event invalidates the current plan (alarm coming from the platform or elaborated by the Robot Monitor function), the supervisor manages the reaction to this event. In some cases it can call the Robot Planner to generate a new plan or repair or adapt the current one, based on the updated current situation of the vehicle.

The Supervisor has full authority to manage the generation and execution of plans ofactions; it may request for a plan generation, start, suspend, resume or abort the execution of a plan.

\section{Planner function}

The Robot Planner module can produce an individual plan of actions for each high level task. Three types of planners are available onboard:

- Area coverage: covering a given area with a given sensor;

- Motion planning: finding the best path(s) from a location to another;

- Resource management (battery, sensors switched on and off...)

This function is not called when actions are directly sent by the MMT, and in this case there is no recomputation of plan at the vehicle level: the disturbing events are globally treated at the MMT level.

Planners in the RSOA correspond to the different tasks identified in underwater operations for the SWARMs project: TRANSIT, INSPECT, SURVEY, PICK_UP. The planner can also be used to build reaction plans to cope with some events. These tasks are SURFACE, REFERENCING, RECOVER and SETMODE. For instance when a loss of positioning precision is detected by the platform vehicle an event is generated and the Supervisor trigger a reaction by asking to the planner to build a SURFACE plan to get a GPS positioning before resuming the ongoing mission plan.

For each of these possible tasks the Robot Planner is able to deliver a plan of actions. For some tasks there are several possible planners. At the moment the choice of the planner to use is specified in the task specification part of the vehicle configuration file.

\section{E. Monitor function}

The Robot Monitor module produces high level health and performance indicators: it monitors the different activities going on at vehicle level and generates events or alarms when unexpected behaviour is detected. The monitor integrates both numeric and qualitative reasoning to provide diagnosis (now) and prognostic (future) for each generic vehicle action. It is therefore directly connected on the one hand to the Robot 
Generic Interface module and on the other hand to the Robot Supervisor module.

The module monitors position, depth, heading and measurements as well as the current action performed and raises alarms when detecting abnormal values. When abnormal values are detected repeatedly, they are escalated into more severe alarms according to a model expressed with temporal logic. The uncertainty about the cause of abnormal values is resolved via a model expressed with conditional preferences. More information is given in [1].

\section{F. Implementation}

All modules of RSOA have been implemented using the Robot Operating System (ROS) framework. Fig. 1 shows the final architecture. This architecture has been implemented in several SWARMs vehicles (mentioned later on in the paper).

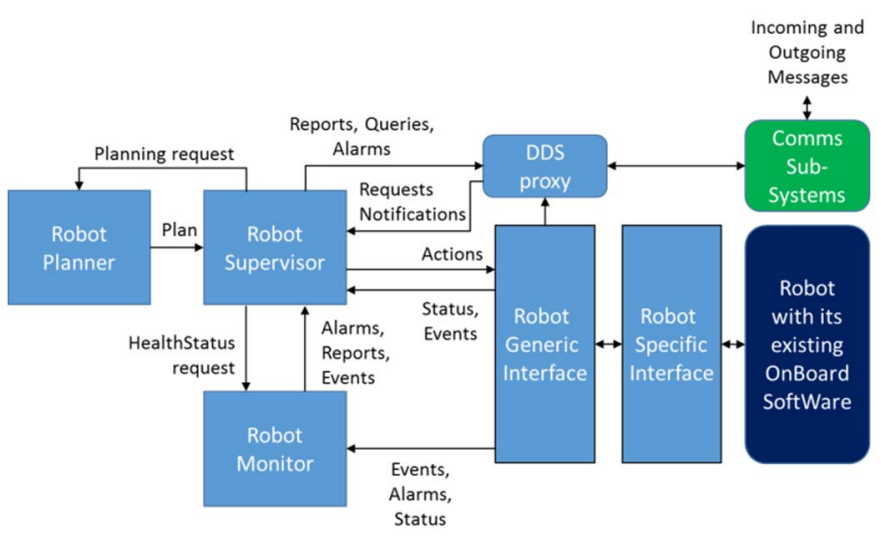

Fig. 1. Modules of the Robot Software Onboard Architecture RSOA.

\section{ROMANIA DEMONSTRATION - JULY 2017}

After early trials in Gran Canaria Island (Spanish) on the Atlantic Ocean in September 2016, the first SWARMs project demonstration took place in Mangalia (Romania) in July 2017, on the Black Sea.

A part of RSOA was implemented in the control station of 2 ROVs and onboard 1 USV. Its whole capabilities were mainly evaluated by simulation.

An Underwater Unmanned Vehicle (UUV) simulator [2] has been developed during the project. It is based on the $3 \mathrm{D}$ open source robotics simulator Gazebo and allows the simulation of environment and vehicle dynamics. Simulations allowed to check the progress of work early in the project, are free from experimental constraints and allow going beyond the experimental capabilities in terms of mission scenario, number of vehicles, type of payload...

Many figures in this paper are representation of the mission using the rviz ROS visualisation tool.

\section{A. Simulation}

The simulated use case was a sonar survey mission performed by a swarm of 8 vehicles (6 AUVs and 2 ROVs) on a $200 \mathrm{~m} \times 200 \mathrm{~m}$ area, each vehicle implementing one RSOA.

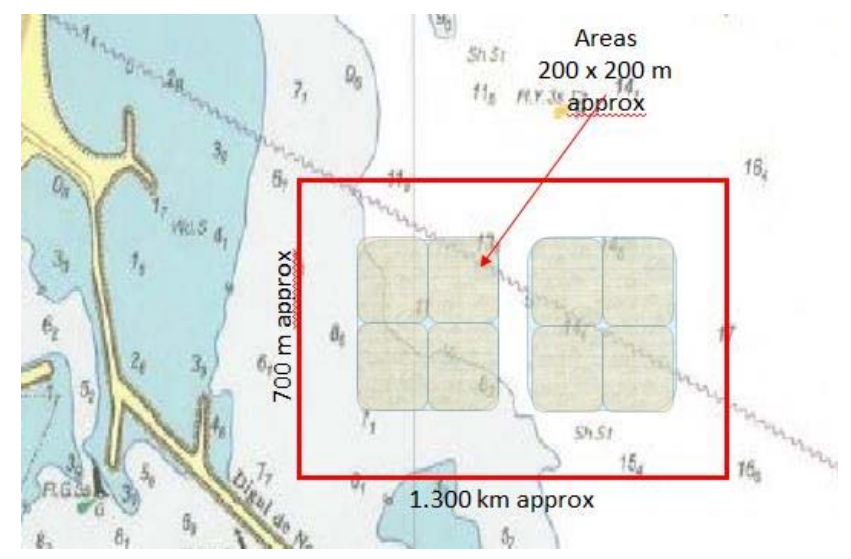

Fig. 2. Simulation mission area in Romania.

The area has then been divided in 8 subareas (Fig. 2). Each vehicle has been assigned by hand to one subarea and several recovery points were available (Fig. 3). There was no connection with the MMT for these tests and a specific mission management system sent the high level survey tasks to vehicles. Once received, the mission started. Each local survey was computed by the Robot Planner; the survey task, sonar scanning along a boustrophedon-shaped trajectory, was translated into a list of "follow row" and "go to waypoint" actions.

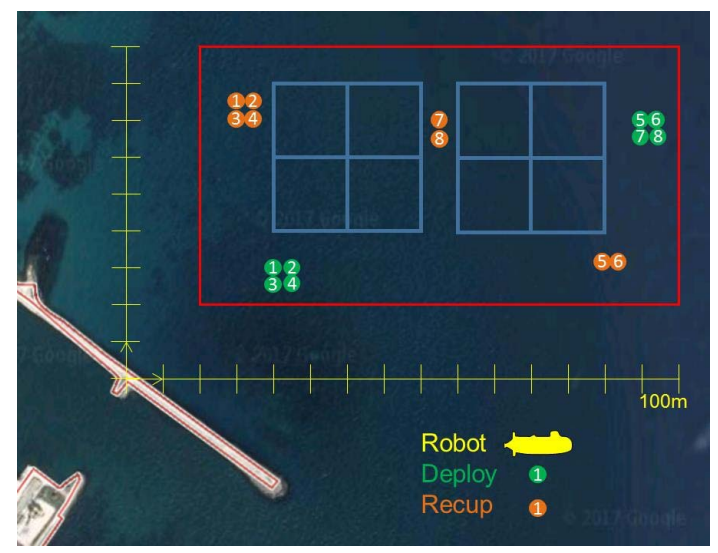

Fig. 3. Simulation mission in Romania.

Several runs highlighted the nominal and reconfiguration capabilities of the architecture:

- Nominal execution of the mission (Fig. 4): basic planning, execution and supervision of actions (including activation and deactivation of payload), storing of robot position, robot status, environment data and robot alarm;

- On line and autonomous adaptation to disruptive events (plan repair): deviation due to a loss of propulsion efficiency under strong currents (Fig. 5), breakdown of one side scan sonar (Fig. 6);

- Recovery operation (Fig. 7): aborting of mission, selection in real time of one recovery area, recovery achievement. 


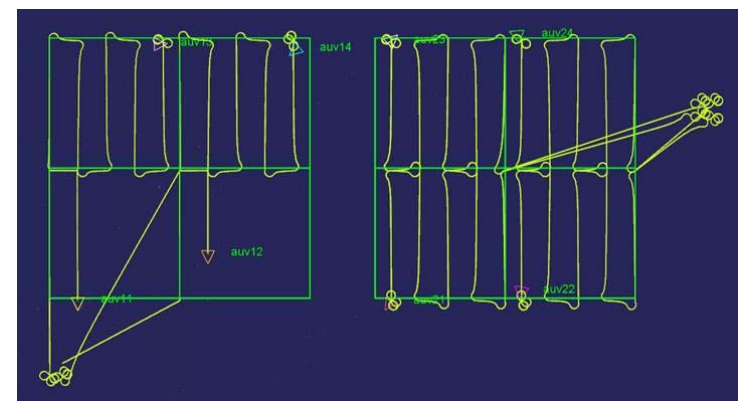

Fig. 4. Simulation of the Romania nominal execution (the 2 ROVs on the left below subareas have a relative lower speed).

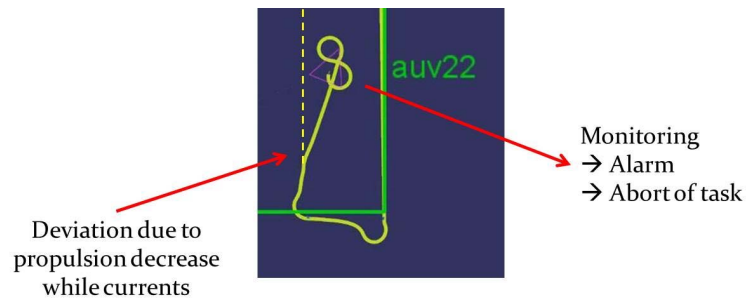

Fig. 5. Reactions to the detection of a strong deviation (auv22 ends its mission by making a kind of hippodrome; the global reaction to send another vehicle to replace it was not done because the MMT was not in the loop).

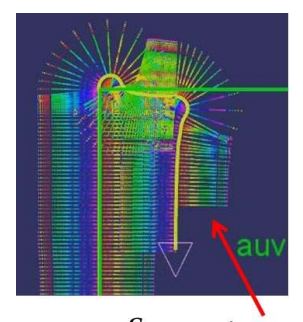

Sonar: stop one side
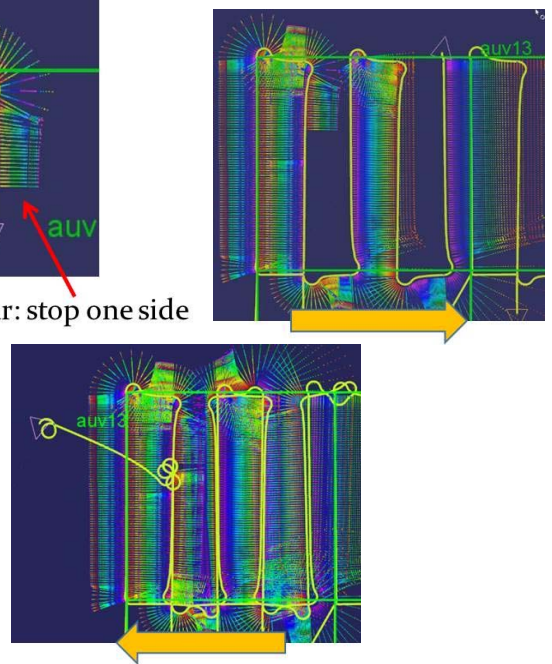

Fig. 6. Reactions to the breakdown of one side of the side scan sonar (the strategy was to continue the same planned rows until the end of the planned survey then to turn back and to follow the same rows in the other sense so as to complete the cover).

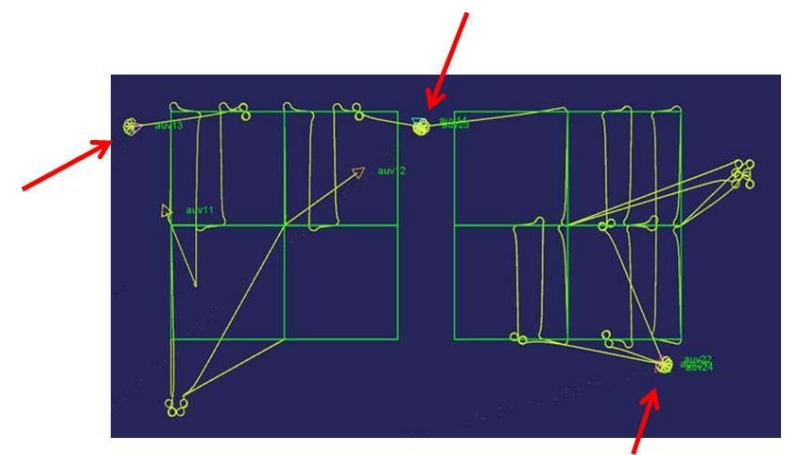

Fig. 7. Recovery operation towards areas indicated with a red arrow and selected on line by the RSOA.

\section{B. Experimentation}

The RSOA has been adapted to 3 experimental vehicles:

- Desistek Robotics SAGA and Autonomous Systems ATN50 ROVs: integration of part of the RSOA in their Command and Control Stations;

- Leonardo Static USV: integration of part of the RSOA in the vehicle. The GPS position was well received and the RSOA managed the three onboard payloads.

\section{NORWAY DEMONSTRATION, JUNE 2018}

The second and final SWARMs project demonstration took place in the Trondheim fjord (Norway) end of June 2018. The use case was to detect and track a plume of traversable pollutant so as to be able to find and act on the leak. The plume was detected by monitoring the water salinity that decreased in the presence of unsalted pollutant. The added capabilities of the RSOA required the enhancement of all modules and the adaptation of functions to the new scenario. The full version of RSOA was integrated in 3 AUVs: Fridtjof LAUV from NTNU and 2 Noptilus LAUVs from Porto University UPTEC. The RSOA was also implemented onboard the Telemetron USV from Maritime Robotics.

The UUV Simulator was enhanced with the objective to simulate the new experimental scenario and especially to model of the Trondheim environment and the vehicles that participated to the experimentation.

\section{A. Norway scenario}

While the Romania simulation scenario was especially built for the evaluation of the RSOA, the Norway simulation scenario (Fig. 8) replicated the complete experimental scenario.

In order to detect the plume, the research area was covered by the 3 AUVs moving at different depths. The size of the whole search area was $400 \mathrm{~m} \times 400 \mathrm{~m}$. All AUVs were deployed far from each edge of the area at a sufficient distance to dive and ascend in safety conditions. The plan computed at the beginning of the mission was computed for each AUV to perform a survey while acquiring salinity data. The USV was used to relay the communication between AUVs and the MMT; it was making circles in the North-East outside the research area to avoid a possible collision as the AUVs could surface anytime. A ROV could then investigate the seabed and act on the leak once its location is known enough. The recovery points were the initial points for each vehicle; the recovery procedure was for each vehicle to join them first at the safety survey depth before going to the surface outside the survey area.

The plume was emulated both in the global simulation and experimentally; the virtual leak was in the North-East of the area and was pushed towards the South and the surface because of diffusion, buoyancy, and a simulated current. 


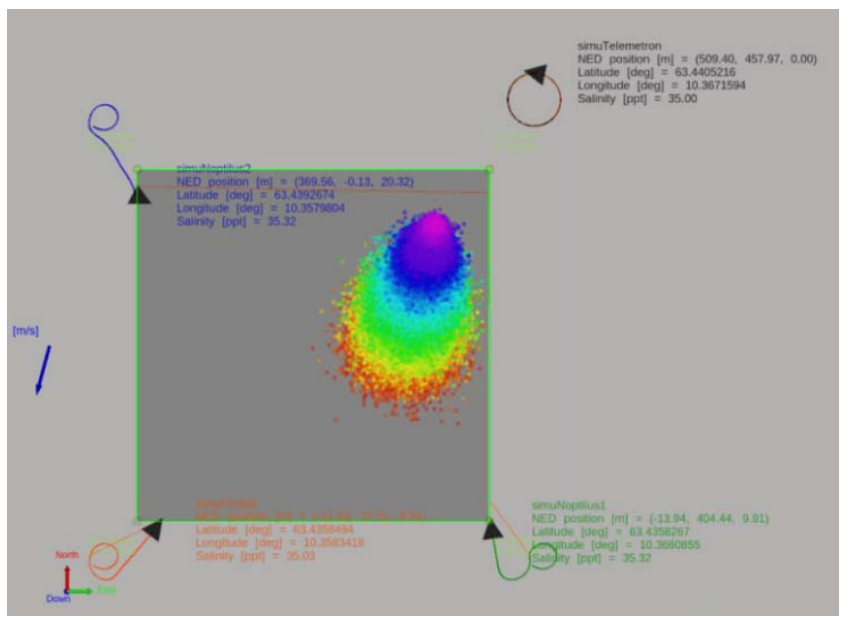

Fig. 8. Simulation mission in Norway: vehicles starting mission and emulated plume. Numbers near each vehicle give their current coordinates.

\section{B. Experimentation}

The sea demonstration showed that all components of the SWARMs system were in the loop: the communication MW, the MMT for the definition of the mission and for the high level planning, the CAF (Context Awareness Framework) to track and map salinity levels, the plume emulator and the RSOAs onboard all vehicles (Fig. 9). At sea, there was no real pollutant and no sensor has been installed on AUVs. The salinity information was sent from the plume emulator to the CAF taking into account the real AUVs positions into account and the AUVs followed their initial planning. A new area could have been assigned to the AUVs after the analysis of the pollution at the MMT level.

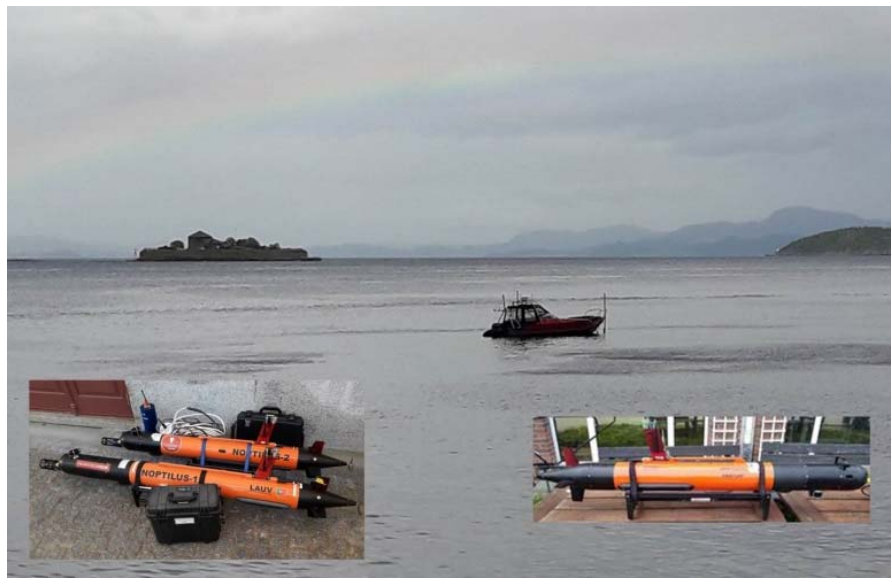

Fig. 9. Vehicles with RSOA in Trondheim.

Above results are given from RSOA point of view implemented in each vehicle:

- Difficulties encountered concerned the implementation of OpenSplice on several processors (Beagle Bone, Raspberry Pi, Jetson); there were some issues on the implementation of DDS on the acoustic channel;

- RSOAs achieved correctly their part of work: reception of high level tasks, computation of relative actions plan, supervision of their execution, collect of data, sending of data to the MMT.

\section{Simulation}

For this scenario, the objective of the onboard strategy was to obtain a maximum of information on the plume so as to find its origin. This was performed thanks to a three-step behaviour coded in the Supervision function and individually followed by each AUV:

- The planner computed a boustrophedon-shaped trajectory in the survey area. Fig. 10 zooms on the beginning of the simulation of the mission for one AUV; the distance between two following rows is about 50 meters.

- A significant decrease, followed by a certain increase of the salinity measurement monitored was interpreted as the the AUV entering then leaving the plume. The ongoing row was then stopped and a U-turn was planned so as to recover the next planned row. This was performed until the end of the initial survey. Fig. 11 shows different U-turn patterns for two AUVs and the evolution of their salinity data.

- After the whole coverage of the initial area, the monitored data of the salinity were used onboard to compute a reduced area and a new SURVEY plan, as shown on Fig. 12.

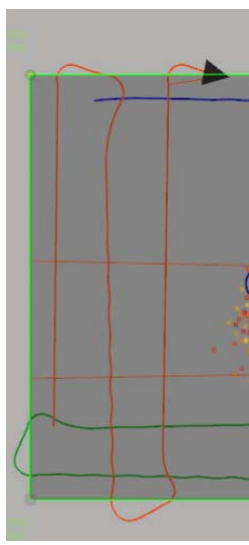

Fig. 10. Rows followed by one AUV.

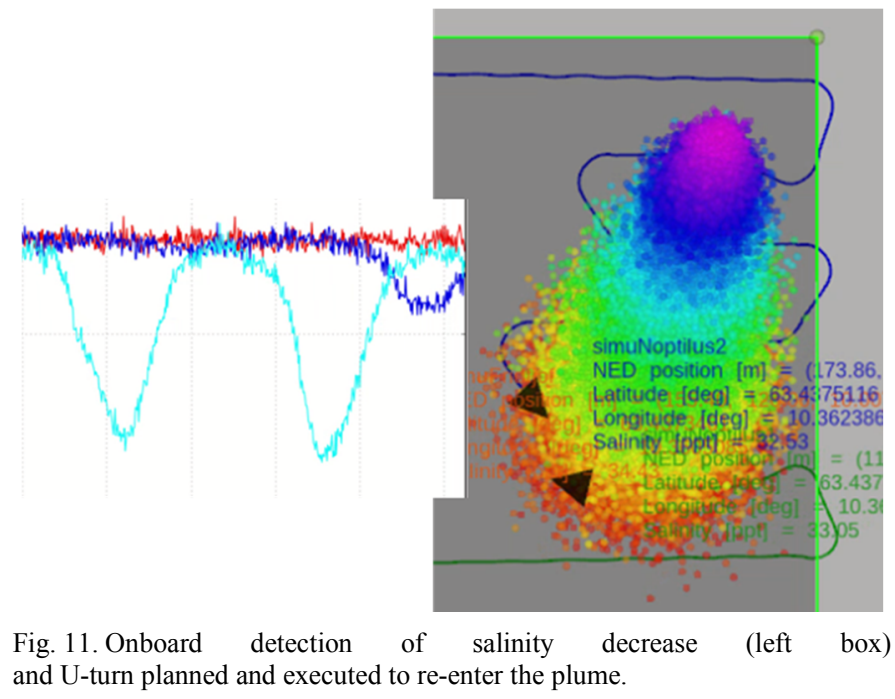

Fig. 11. Onboard detection of salinity decrease (left box) 


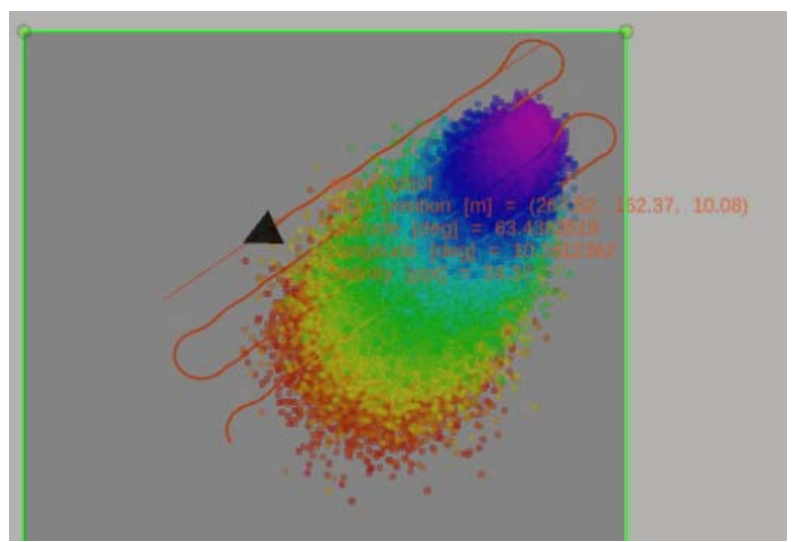

Fig. 12. Autonomous planning and execution of a new survey to better track the plume.

Fig. 13 highlights the capability onboard each vehicle to compute a safe recovery plan towards the starting area.

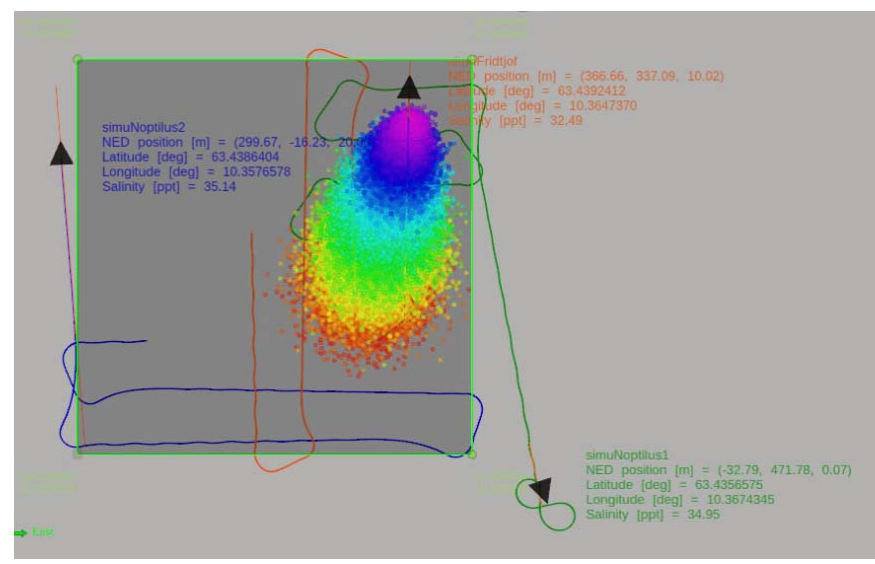

Fig. 13. Recovery at the end of the initial survey.

\section{CONCLUSIONS AND PERSPECTIVES}

A generic and modular architecture for maritime autonomous vehicles, named RSOA, has been developed and tested both by simulation and at sea during the SWARMs H2020 project. Its development was incremental and applied in two different scenarios: sonar survey of an area and detection and tracking of a plume of pollutant.

The final demonstration in June 2018 highlighted the good behaviour of the RSOA. The choice of the ROS open source framework allowed the development by several project partners and the use of different planners. The modularity also facilitated the integration in several types of uninhabited autonomous vehicles and even in control stations of semiautonomous teleoperated vehicles. The genericity of the architecture is proved by these various integrations in heterogeneous robots.

Simulations and demonstrations highlighted the below capabilities of SWARMs vehicles (AUVs, USVs and ROVs):

- Completion of high level tasks thanks to onboard planning capabilities;

- Information exchange with the high level MMT;
- Online and onboard adaptation of the mission when occurrence of disturbing events while using associated specific behaviours.

Perspectives concern first the enhancement of onboard functions at one vehicle level; this was difficult during the project life because first implementation on real vehicles took a lot of time and second the simulator had only real time simulation capabilities.

- More planners were developed but not tested as the plume scenario was relatively simple;

- A generic model of replanning strategies to face disruptive events could have been used, for example a mix between a state automata and a resources scheduler;

- The robustness of the monitoring function has not been tested.

Perspectives concern also the direct cooperation in the team of robots. Indeed, all cooperation between vehicles in the SWARMs project was through the centralized MMT: either the reaction was local at the vehicle level or the reaction was global at the MMT level. Global strategies could be developed like those ones evaluated for the survey of an area by a team of aerial and terrestrial vehicles in the ACTION project [3]. The difficulty remains in the direct communication between robots for this cooperation, depending on the sensors, the perception function and physical characteristics of the environment. A solution could be obtained by communication via the MMT as it can forward to vehicles information about the other ones.

\section{ACKNOWLEDGMENT}

This work was partially funded by the SWARMs European Project (Smart and Networking Underwater Robots in Cooperation Meshes), under Grant Agreement No. 662107SWARMs-ECSEL-2014-1.

The authors thank the project partners for their participation to the RSOA work and its evaluation, and especially: S. Bilbao, Tecnalia, Spain, F. Pacini, Leonardo, Italy, J. Dreo, Thales R\&T, France, M.M.M. Manhaes, Robert Bosch GmbH, Germany. Only results from author's developments are shown in this paper.

\section{REFERENCES}

[1] X. Pucel and S. Roussel (2017). Intermittent Fault Diagnosis as Discrete Signal Estimation: Trackability analysis. Prooceedings of the 28th international Workshop on Principles of Diagnosis (DX'17).

[2] M. M. M. Manhães, S. Scherer, M. Voss, L. R. Douat and T. Rauschenbach, "UUV Simulator: A Gazebo-based Package for Underwater Intervention and Multi-Robot Simulation," OCEANS 2016 MTS/IEEE Monterey, Monterey, CA, USA, 19 - 23 Sep. 2016.

[3] P. Bechon, M. Barbier, Ch. Lesire, G. Infantes, and V. Vidal: "Using hybrid planning for plan reparation". ECMR'15, European Conference on Mobile Robots, September 2-4, 2015, Lincoln (UK). 